\title{
Bosques ribereños del trópico húmedo de México: un caso de estudio y aspectos críticos para una restauración exitosa
}

\section{Riparian forest of the humid tropics of Mexico: A study case and critical as- pects for their successful restoration}

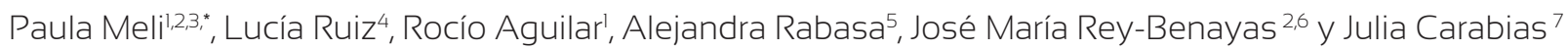

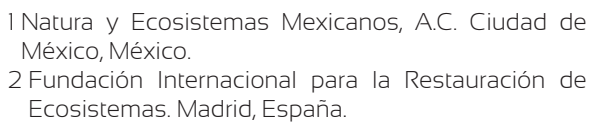

1 Natura y Ecosistemas Mexicanos, A.C. Ciudad de México, México.

2 Fundación Internacional para la Restauración de Ecosistemas. Madrid, España.

\author{
3 Universidad de São Paulo. Piracicaba, Brasil. \\ 4 Comisión Nacional de Áreas Naturales Protegidas. \\ Ciudad de México, México. \\ 5 Consultora Independiente. Ciudad de México, México.
}

\author{
6 Universidad de Alcalá. Madrid, España \\ 7 Universidad Nacional Autónoma de México. Ciudad \\ de México, México. \\ * Autor de correspondencia. atajacaminos@yahoo.com
}

\section{RESUMEN}

Los bosques ribereños albergan una biodiversidad importante y en ellos se desarrollan funciones ecológicas esenciales que repercuten en la provisión de servicios ecosistémicos relevantes para la sociedad. Las perturbaciones de origen antrópico frecuentemente exceden la resiliencia natural de estos ecosistemas y, en consecuencia, desencadenan procesos de degradación. La restauración ecológica busca recuperar la biodiversidad y los servicios de los bosques ribereños, pero su éxito depende de varios aspectos de contexto. En este trabajo se utiliza un estudio de caso para ejemplificar la problemática de la restauración desde una perspectiva 'integral' y se discuten aspectos ecológicos, socio-económicos y legales que pueden influir en el éxito de la restauración de estos bosques. Si bien los aspectos ecológicos han sido considerablemente desarrollados, aún falta información relacionada con las limitaciones económicas (p. ej. los costos de producción), sociales (p. ej. la aceptación social) y legales (p.ej. la incorporación de la restauración ecológica en programas públicos), para ser aplicados a escalas más amplias. La estimación de los servicios ecosistémicos que podrían restablecerse a partir de la restauración de bosques ribereños aportará a la integración de estos aspectos. El abordaje de estos aspectos a escala local aportará información para el desarrollo futuro de estrategias a escala regional más eficientes en términos de la relación beneficio ecológico-costo socioeconómico. Se discuten algunas recomendaciones relacionadas con la conservación, el manejo y la restauración de los bosques ribereños en el trópico húmedo de México.

PALABRAS ClAVE: marco legal, percepción social, recuperación, ribera, selva, socioeconómico.

\section{ABSTRACT}

Riparian ecosystems harbor significant biodiversity and support essential ecological functions that affect ecosystem services provision for human well-being. Anthropogenic disturbances often exceed the natural resilience of these ecosystems thus resulting in their degradation. Ecological restoration promotes restoring biodiversity and riparian ecosystems services, but its success depends on several aspects. This work bases on a study case to exemplify the problem of restoration from an 'integral' perspective and discusses ecological, socio-economic and legal aspects that can influence the success of the restoration of these forests. While ecological aspects have been considerably developed, information on economic (e.g. production costs), social (e.g. social acceptance) and legal constraints (e.g. incorporation of restoration in public programs) are still limited for restoration being applied at broader scales. Estimating ecosystem services that could be restored from the restoration of riparian forests may help to integrate these aspects. Addressing these aspects at the local level will provide information for the future development of more efficient regional strategies in terms of the ecological benefit-socioeconomic cost ratio. Finally, we discuss some recommendations relating to the conservation, management and restoration of riparian ecosystems in the humid tropics of Mexico.

KEY WORDs: legal framework, social perceptions, recovery, riparian, rainforest, socio-economic. 


\section{INTRODUCCIÓN}

Los bosques ribereños son sistemas complejos y únicos en términos de sus características biofísicas y procesos ecológicos, dado que constituyen una interfase entre los ecosistemas terrestres y los acuáticos. Estos bosques contribuyen al albergar una alta diversidad de especies vegetales y animales, así como al sostener numerosos procesos biológicos; siendo esta contribución muchas veces proporcionalmente mayor a la superficie que ocupan dentro de una cuenca (Sabo et al., 2005). En México, la condición ecológica de los bosques ribereños a escala nacional nunca ha sido evaluada, pero a escalas locales, y en particular en el trópico, algunos estudios han demostrado los altos niveles de degradación y transformación que estos ecosistemas han sufrido y los impactos que esto puede tener no solo a nivel ecológico (Ramírez-Martínez, 2010), sino también sobre el bienestar de las poblaciones locales asociadas a ellos (Allen, Santana-Michel, Ortiz Arrona y Zedler, 2010; Meli, Hernández-Cárdenas, Castro y Carabias, 2015c). En este contexto, resulta de crucial importancia generar información pertinente y adecuada para promover la restauración de estos ecosistemas.

Tradicionalmente la restauración ecológica se ha definido como el proceso de ayudar al restablecimiento de un ecosistema que ha sido degradado, dañado o destruido (Society for Ecological Restoration , 2004). Sin embargo, en los últimos años la restauración ha emergido como una alternativa para promover un nuevo paradigma de un desarrollo socioeconómico mejor integrado con la naturaleza (Echeverría, Smith-Ramírez, Aronson y BarreraCataño, 2015), que aporta a la conservación de la biodiversidad y al mismo tiempo mejora el bienestar social (Suding et al., 2014; Mc Donald, Gann, Jonson y Dixon, 2016). Desde esta perspectiva, abordar la restauración únicamente desde sus aspectos ecológicos resultaría incompleto. Una estrategia de restauración debe integrar información sobre aspectos ecológicos, sociales y legales (Budiharta, Meijaarda, Wells, Abram y Wilson, 2016), así como considerar la relación beneficio-costo que resulta de sus acciones (Kimball et al., 2015). En América Latina, por ejemplo, aunque los aspectos técnicos de la restaura- ción han sido bien desarrollados, es muy reciente la consideración de la dimensión social para guiar la restauración y su potencial para satisfacer las necesidades humanas (Ceccon y Pérez, 2016). Son escasas las evaluaciones integrales de los aspectos ecológicos, sociales y legales de la restauración de bosques (Budiharta et al., 2016). El presente trabajo aborda algunos aspectos que emergen de la problemática de la restauración de bosques ribereños en México con especial énfasis en los trópicos húmedos. El objetivo principal de este estudio es aportar información pertinente para la toma de decisiones en el manejo y restauración de estos ecosistemas en cuanto a sus aspectos ecológicos, legales y socio-económicos. El trabajo no describe ni analiza la restauración de los ecosistemas ribereños en general, sino que utiliza un estudio de caso para ejemplificar la problemática de la restauración desde una perspectiva 'integral' y discute algunos aspectos que pueden influir en el éxito de la restauración de estos bosques. También se discuten algunas implicaciones de la restauración de bosques ribereños a escala regional y, finalmente, se proponen algunas recomendaciones sobre estos aspectos de la restauración de estos ecosistemas.

\section{Estudio de caso: Marqués de Comillas}

El municipio Marqués de Comillas se encuentra en el sur del Estado de Chiapas. Posee una superficie total de 94 315 hectáreas y se compone de 27 ejidos, con una población total de un poco más de 9500 personas (Instituto Nacional de Estadística y Geografía, 2010). Su relieve constituye una serie de lomeríos con llanuras, con altitudes máximas de $220 \mathrm{~m}$ snm, con clima cálido húmedo y subhúmedo, precipitación media anual de $2143 \mathrm{~mm}$ y temperatura media anual entre $22{ }^{\circ} \mathrm{C}$ y $26^{\circ} \mathrm{C}$. Existe una gran diversidad de suelos y considerable variación topográfica, así como una compleja red fluvial que determinan las selvas alta y mediana perennifolias como los tipos de vegetación más sobresalientes (Instituto Nacional de Ecología, 2000). Su paisaje actual constituye una matriz agropecuaria que incluye importantes fragmentos remanentes de selva pero también grandes áreas deforestadas que incluyen a los bosques ribereños, lo que determina altera- 
ciones importantes en los cauces y en las poblaciones de fauna acuática (Ramírez-Martínez, 2010).

En el año 2012, en el municipio se llevó a cabo el "Ordenamiento comunitario del territorio de la microrregión Marqués de Comillas, Chiapas”, dentro del cual se planteó la necesidad de establecer y poner en marcha acciones de restauración ecológica, particularmente de los bosques ribereños (Gaia y Natura y Ecosistemas Mexicanos A.C. [Natura], 2012; Castro y Ortiz, 2015). Este programa promovió un proceso microregional de varios ejidos que facilitó la integración de acciones dentro de un esquema territorial más amplio, la conjunción de acciones colectivas e institucionales de mayor amplitud y una dinámica social de mayor capacidad para la regulación y la gestión territorial. En el marco de este programa y desde algunos años anteriores a este, se desarrollaron estudios de distinta índole para abordar la problemática de la restauración de bosques ribereños en este municipio. Estos trabajos son mencionados a lo largo de este texto, y en ellos se basa el mismo.

\section{Aspectos críticos para la restauración de bosques ribereños en México}

\section{Aspectos ecológicos: especies y manejo de las riberas degradadas}

Históricamente la restauración implicaba un retorno a las condiciones del ecosistema original, definido como de 'referencia', pero bajo las actuales condiciones de rápido cambio ambiental y cultural se ha cuestionado la relevancia de definir este ecosistema para aceptar múltiples trayectorias potenciales para los ecosistemas, enfatizando el proceso de restauración para abarcar objetivos que sean pragmáticos para el bienestar humano (Higgs et al., 2014) y socioeconómicamente viables (Suding et al., 2014). En el trópico húmedo de México la vegetación ribereña presenta una gran variación en su gradiente longitudinal y lateral (i.e. desde el cuerpo de agua y hacia el interior del bosque), lo que determina que los procesos fluviales y el régimen hidrológico sean muy específicos para cada sitio (Ramírez-Carrillo, 2006). En este contexto, sería más útil definir 'condiciones de referencia' (e. g., composición, estructura, procesos) en lugar de un ecosistema particular. Esto permitiría definir de manera más clara los objetivos de la restauración y los indicadores que serán utilizados para medir su éxito, aunque aún falta valorar con mayor detalle el ámbito de variación crítico de estas condiciones para lograr el restablecimiento de las funciones y procesos ecológicos de los bosques ribereños.

Una de las necesidades principales en las áreas deforestadas es el restablecimiento de la cobertura arbórea, que tendrá funciones múltiples como disminuir la erosión y favorecer la retención de sedimentos, recuperar condiciones ambientales más favorables para el restablecimiento de las especies características de estos bosques o la atracción de dispersores de semillas (Meli, Martínez-Ramos, Rey Benayas y Carabias, 2013). La riqueza de especies arbóreas nativas de uso potencial para la restauración en los trópicos es elevada (Meli, Martínez-Ramos, Rey Benayas y Carabias, 2014), por lo que no debería limitarse a un número reducido de especies. Sin embargo, trabajar con un gran número de especies requiere un tiempo y esfuerzo que, en la mayoría de los casos, no es posible invertir, por lo que la identificación de especies clave es de gran utilidad, sobre todo si existen expectativas de implementar acciones de restauración a mediana o gran escala.

En Marqués de Comillas, de un listado de más de 100 especies arbóreas presentes en las riberas se identificaron 30 que resultan clave y que: (1) son importantes en el ecosistema de referencia, (2) requieren de acciones de restauración activa (i.e. ser introducidas), (3) tienen algún valor social y (4) son relativamente fáciles de propagar (Tabla 1). Esta cifra no incluye la gran cantidad de especies que son típicamente poco abundantes en estos bosques, las cuales deberán ser evaluadas y eventualmente introducidas en etapas posteriores (Meli, Martínez-Ramos y ReyBenayas, 2013). Además, el establecimiento de estas primeras especies clave no garantiza la llegada de especies del sotobosque (Holl y Crone, 2004), por lo que también es importante considerar la incorporación de especies arbustivas, palmas y herbáceas (Weisberg, Mortenson y Dilts, 2013). En casos de degradación severa, donde no 
Tabla 1. Especies leñosas clave seleccionadas como potencialmente útiles para la restauración de bosques ribereños en Marqués de Comillas, Chiapas.

\begin{tabular}{|c|c|c|c|c|c|c|}
\hline Especie & $D$ & PRN & $H$ & VS & $T c$ & ISS \\
\hline Dialium guianense & 8 & 10 & 9 & 9 & 7 & 43 \\
\hline Brosimum alicastrum & 6 & 6 & 9 & 9 & 8 & 38 \\
\hline Brosimum costarricanum & 6 & $10^{*}$ & 7 & 6 & 8 & 37 \\
\hline Ficus sp. & 10 & 9 & 2 & 8 & 7 & 36 \\
\hline Cojoba arborea & 10 & 6 & 4 & 5 & 7 & 32 \\
\hline Vochysia guatemalensis & 5 & 7 & 7 & 8 & 5 & 32 \\
\hline Trophis racemosa & 4 & $10^{*}$ & 6 & 6 & 6 & 32 \\
\hline Albizia leucocalyx & 5 & 8 & 3 & 8 & 7 & 31 \\
\hline Ampelocera hottlei & 6 & 3 & 7 & 6 & 9 & 31 \\
\hline Calophyllum brasiliense & 5 & 6 & 7 & 6 & 7 & 31 \\
\hline Licania platypus & 5 & $10^{*}$ & 6 & 6 & 4 & 31 \\
\hline Posoqueria latifolia & 5 & $10^{*}$ & 6 & 5 & 5 & 31 \\
\hline Guarea glabra & 5 & 3 & 7 & 6 & 8 & 29 \\
\hline Protium copal & 7 & 3 & 9 & 6 & 3 & 28 \\
\hline Castilla elastica & 5 & 3 & 6 & 6 & 7 & 27 \\
\hline Hirtella americana & 4 & 4 & 7 & 5 & 7 & 27 \\
\hline Pouteria durlandii & 5 & 5 & 7 & 6 & 4 & 27 \\
\hline Swartzia simplex & 5 & $10^{*}$ & 3 & 5 & 4 & 27 \\
\hline Blepharidium mexicanum & 4 & 5 & 6 & 4 & 7 & 26 \\
\hline Inga vera & 4 & 5 & 3 & 9 & 5 & 26 \\
\hline Eugenia negrita & 4 & $10^{*}$ & 7 & 0 & 5 & 26 \\
\hline Quararibea yunckerii & 4 & $10^{*}$ & 3 & 6 & 3 & 26 \\
\hline Nectandra reticulata & 5 & $10^{*}$ & 6 & 0 & 4 & 25 \\
\hline Miconia argentea & 4 & 5 & 4 & 6 & 5 & 24 \\
\hline Jacaratia dolichaula & 4 & $10^{*}$ & 6 & 0 & 4 & 24 \\
\hline Croton schiedeanus & 5 & 2 & 6 & 5 & 5 & 23 \\
\hline Eugenia mexicana & 5 & 6 & 4 & 0 & 5 & 20 \\
\hline Licaria capitata & 4 & $10^{*}$ & 4 & 0 & 2 & 20 \\
\hline Nectandra sanguinea & 5 & $10^{*}$ & 1 & 0 & 4 & 20 \\
\hline Miconia glaberrima & 4 & $10^{*}$ & 1 & 0 & 3 & 18 \\
\hline
\end{tabular}

El índice de selección de especies (ISS) integra valores estandarizados de la dominancia natural (D), el potencial de regeneración natural (PRN), la amplitud de hábitat (H), el valor social (VS) y limitaciones técnicas para propagar la especie (TC). (*) Especies ausentes en bosques ribereños secundarios que deberían ser consideradas para su reintroducción (valores altos de NRP). Fuente: Meli et al. (2014). 
hay cobertura vegetal y se ha perdido parte del suelo, podrían ser útiles aquellas especies con alta densidad de raíces, que favorecen la retención de sedimentos y el control de la erosión por el agua del borde de los cauces, como son la caña brava (Phragmites australis) y la jimba (Bambusa longifolia).

Considerar el valor social y cultural de las especies también es crítico para lograr una restauración exitosa (Hobbs et al., 2004) (ver apartado aspectos socioeconómicos). En algunos casos aquellas especies que caracterizan al bosque maduro no son las especies con mejor desempeño (i.e. supervivencia y crecimiento) en el campo, pero suelen ser las más valoradas por las poblaciones locales (Meli et al., 2014). Estas especies nativas no deberían ser excluidas de los esfuerzos de restauración, sino que deberían buscarse estrategias para mejorar su desempeño. Una alternativa sería su introducción en etapas posteriores, utilizando la facilitación por parte de las especies de etapas tempranas o sombra artificial.

Desde los aspectos ecológicos, el nivel de intervención sobre las riberas degradadas puede determinar distintos tiempos de recuperación. La restauración pasiva (i.e. detener el disturbio para permitir la recuperación natural o no asistida), suele ser la estrategia más adecuada, ecológica y económicamente, sobre todo cuando el proceso de regeneración natural puede ocurrir una vez que los factores de degradación son eliminados. En este caso, el estudio de la composición específica de la vegetación de las riberas degradadas resulta valioso para evaluar aquellas especies o procesos que podrían restablecerse de forma pasiva. En Marqués de Comillas, como en otras regiones tropicales, la regeneración natural y el desarrollo de selvas secundarias ocurre cuando los terrenos productivos son abandonados. Pero la composición de especies de la selva secundaria es diferente a la observada en la selva madura (MartínezRamos, Barraza y Balvanera, 2012): en la primera existe abundancia de arbustos y árboles de bajo porte (MartínezRamos y García-Orth, 2007). Esto que sugiere que algunas especies no logran establecerse y que en estas selvas secundarias no necesariamente se restablecen algunas funciones ecológicas.
La restauración activa supone una elevada gama de intervenciones humanas que tienen el fin de influir en la trayectoria sucesional de la recuperación, particularmente de acelerarla, .pero esto implica mayores costos (ver aspectos socioeconómicos). Considerar ambas opciones es importante para valorar la relación beneficio-costo de cada una de ellas y para determinar los sitios adecuados para aplicar cada una de estas estrategias (Watanabe et al., 2005). Una estrategia combinada podría ser la más exitosa: favorecer la restauración pasiva de algunas especies (p. ej. favorecer el crecimiento de las especies que se establecen de forma pasiva) y, al mismo tiempo, introducir activamente aquellas especies que no se establecerán por sí solas (Meli, Martínez-Ramos y Rey-Benayas, 2013) o, que de no introducirlas, no se detona el proceso sucesional (p.ej. 'liberación de especies', reactivación del banco de semillas, generación de claros, sombreados artificiales). Esto permitiría conocer los posibles efectos 'facilitadores' de las especies que se regeneran naturalmente, en el establecimiento de otras especies, ampliando así el conocimiento para el manejo del bosque ribereño a largo plazo (Rodrigues, Lima, Gandolfi y Nave, 2009).

Cuando la restauración implica la introducción de especies, la historia de uso del sitio determina la condición ambiental que influye en la supervivencia y el crecimiento de las plantas introducidas (Román-Dañobeytia, LevyTacher, Aronson, Ribeiro-Rodrigues y Albores, 2012). Por ejemplo, la llegada de semillas de especies de la selva madura se restringe a aquellas áreas colindantes con los remanentes de selva, y aunque exista lluvia de semillas, el establecimiento de algunas especies puede estar impedido por factores abióticos y bióticos (Martínez-Ramos y García Orth, 2007).

La compactación del suelo, típica de zonas ganaderas, no parece constituir un factor crítico que limite la supervivencia de plántulas introducidas en riberas degradadas por la actividad ganadera (Meli, Aguilar-Fernández y Carabias, 2015a). Al contrario, la roturación del suelo podría debilitar el enraizado, haciendo a las plantas más susceptibles de ser arrastradas por la creciente de los ríos en las temporadas de lluvias. De manera paralela, la com- 
petencia con los pastos puede reducir el establecimiento de algunas especies. Si bien se ha observado que estos dos factores pueden funcionar como factores limitantes en los trópicos, en Marqués de Comillas parecen ser de importancia relativamente menor en comparación con el corto pero intenso período de sequía y con el desborde de los arroyos durante la temporada de lluvias (Meli et al., 2015a). En este contexto, los esfuerzos de restauración deberían enfocarse en aumentar la supervivencia más que el crecimiento de las plantas introducidas. La roturación no debería realizarse a menos que la compactación fuera extrema, o bien debería ser combinada con otras técnicas como la adición de una capa de mantillo o acolchado y el riego puntual en el estiaje para evitar la desecación. De manera paralela, la remoción de pastos debería aplicarse solamente durante la época de lluvias y únicamente en caso de que estos superaran la altura de las plantas introducidas (Fig. 1).

El tiempo y el costo asociados a distintas técnicas de restauración puede ser un impedimento para su adopción por parte de las poblaciones locales (Wilson, Jansen, Curtis y Robertson, 2006), por lo que también son necesarias las valoraciones económica y de aceptación social de las herramientas de restauración (ver aspectos socioeconómicos). Un estudio que incluyera varias estrategias de una manera combinada aportaría información pertinente y sólida para la toma de decisiones. Por ejemplo, combinar la aplicación de protecciones para evitar la desecación, mejorar la fertilidad, introducir bancos de semillas, favorecer el crecimiento de las especies leñosas que se establecen de manera natural, utilizar protectores y eliminar pastos solamente en la época de lluvias, entre otras medidas. También sería altamente recomendable el monitoreo de los impactos de estas actividades sobre la fauna silvestre; existen algunas evidencias al respecto en la región (Martínez-Ramos et al., 2012).

\section{Aspectos socioeconómicos}

Cuando se pone en marcha la restauración los aspectos sociales resultan especialmente importantes en regiones socioculturalmente heterogéneas (Budiharta et al., 2016).
Las diferencias entre la percepción de los distintos actores sociales sobre el valor ecológico y la condición 'natural' o 'ideal' de un bosque pueden dificultar la toma de decisiones. Por ello es de vital importancia favorecer el consenso y por ende el éxito de la restauración. Si bien en Marqués de Comillas se percibe una cierta degradación de los bosques ribereños, la magnitud de los efectos negativos asociados con la deforestación no ha sido adecuadamente valorada (Meli, Landa, López-Medellín y Carabias, 2015d). Por esta razón, es recomendable impulsar más programas de recuperación de riberas acompañados por otros de educación ambiental para difundir la importancia de conservarlas y por mecanismos de participación que les permitieran adoptar los procesos de restauración.

También resulta importante considerar las percepciones sociales sobre la capacidad y adecuación de las especies con fines de restauración. Si la diversidad de especies es alta y el conocimiento sobre ellas es limitado, es importante considerar el uso de especies con algún valor social especialmente cuando estas especies motivan a las poblaciones locales a la restauración (Allen et al., 2010). La inclusión de las comunidades locales en la selección de especies es de gran relevancia si se tiene en cuenta que en México, aunque las zonas ribereñas están sujetas a las leyes federales, finalmente las decisiones sobre su uso las

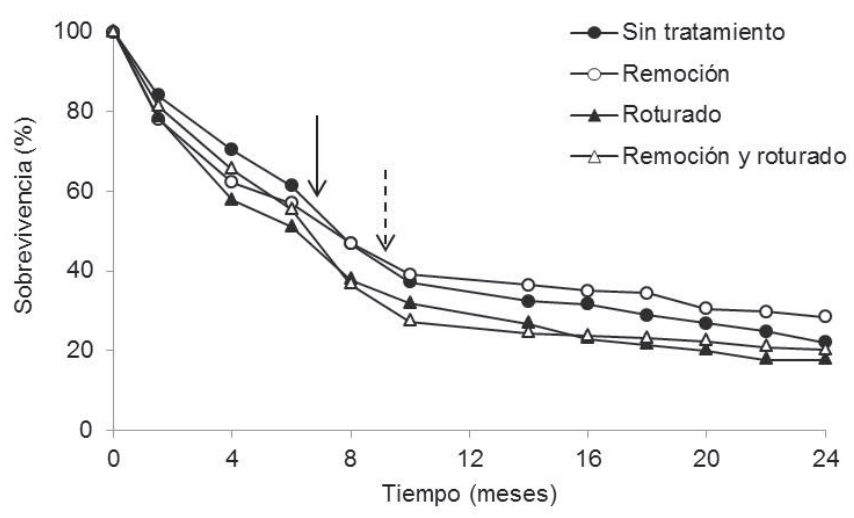

Figura 1. Supervivencia de siete especies arbóreas trasplantadas a riberas degradadas con cuatro técnicas de restauración.

Las flechas indican los eventos de sequía (línea continua) y de crecientes (línea discontinua) de los arroyos. Fuente: Meli et al. (2015b). 
toman los campesinos (Ruiz, 2011). Esto destaca la necesidad de enmarcar la restauración en un contexto social y económicamente aceptable, que incluya las necesidades ecológicas y económicas de los pobladores locales.

Desde los aspectos socioeconómicos, elegir entre un enfoque de restauración pasiva o activa supondría una relación beneficio-costo diferente. Una valoración económica preliminar estimó los costos de una restauración pasiva en Marqués de Comillas en MXN \$14 200 por hectárea (costos del año 2012; Aguilar-Fernández, 2013), lo que podría representar un costo total MXN \$1138 para una sección de ribera de $100 \mathrm{~m} \times 8 \mathrm{~m}$. En el caso de una restauración activa, el costo de producción de una planta en viveros rústicos de la región ronda los MXN \$0.82 (Natura, 2013). Sin embargo, dado que la restauración de riberas incluye además la preparación del terreno (i.e. cercado, tratamiento del suelo y de la vegetación remanente) y el mantenimiento de la plantación, esta misma sección de ribera con una densidad de 0.25 plantas $/ \mathrm{m}^{2}$ costaría MXN \$5300 en un periodo de dos años (Natura, 2013). Este costo equivale a 4.3 veces un salario mínimo mensual en México (Comisión Nacional de Salarios Mínimos, 2015) y equivale a la cuarta parte de los ingresos anuales de un campesino en la región (Centro Interdisciplinario de Biodiversidad y Ambiente, 2010), por lo que resulta demasiado elevado para ser asumido por un productor. En estos casos, se podrían decretar áreas de protección forestal y compensar a los propietarios con estímulos económicos como el pago por servicios ambientales, además de los ingresos que recibirían por el aprovechamiento de las especies útiles (Natura, 2013) o desgravación fiscal (Bullock, Aronson, Newton, Pywell y Rey Benayas, 2011). Estos instrumentos pueden ser una alternativa para la conservación y el manejo sustentable de estos bosques basados en una clara distribución de competencias y una coordinación institucional (ver aspectos legales), pero para ello es imprescindible claridad en los regímenes de tenencia de la tierra.

Las actividades de restauración podrían ser incluidas en programas gubernamentales a distintas escalas (federales o estatales), orientados a la conservación y manejo de los recursos forestales o acuáticos (Meli et al, 2015a). En México, una experiencia reciente fue el Programa Especial para la Conservación, Restauración y Aprovechamiento Sustentable de la Selva Lacandona, en el Estado de Chiapas (PESL), que operó la Comisión Nacional Forestal entre 2010 y 2015 (Meli et al., 2015a) como un apartado especialmente orientado a la región en el marco del Programa Nacional Forestal (Pronafor). Sus lineamientos distinguieron la restauración de riberas de la reforestación diversificada (aquella que se efectúa en la periferia de los remanentes de vegetación o incluso en áreas aisladas, transformadas en su totalidad). Este programa constituyó una línea base en la preocupación de las instituciones y las comunidades locales por la restauración de riberas y por ajustarse a las características específicas de la región. Esto resulta particularmente importante considerando la proyección que los dueños pueden hacer sobre el uso futuro de sus parcelas.

\section{Aspectos legales}

La conservación y restauración de los bosques ribereños dependen tambien de los aspectos legales que regulan la propiedad y el uso de la tierra. Desafortunadamente, en América Latina, excepto en países como Brasil (SoaresFilho et al., 2014) o Costa Rica (Ortiz, Rubio, Carabias y Vázquez, 2015), los bosques ribereños no suelen ser considerados como ecosistemas en los programas gubernamentales ni en las políticas públicas relacionadas con el manejo de recursos. México no es la excepción, y actualmente en su marco jurídico no existe ningún instrumento diseñado específica o explícitamente para la conservación de estos ecosistemas con una perspectiva de ecotono, ni que defina una anchura mínima de la franja de conservación para el mantenimiento de su biodiversidad y sus funciones ecológicas (Ruiz, 2011; Meli et al., 2015a). Sin embargo, existen algunas disposiciones que son aplicables a su conservación, manejo y restauración.

El marco de referencia más general se encuentra en el párrafo quinto del artículo 27 de la Constitución, que determina a las aguas nacionales bajo un régimen de propiedad pública. A su vez, la Ley de Aguas Nacionales 
reglamenta este párrafo y define una franja ribereña como 'zona federal', desde un enfoque operativo y no ecológico (Tabla S1). En este contexto, todos los bosques ribereños estarían asociados a cuerpos de agua que constituyen un bien público, por lo que deberían ser considerados como tales. En el marco de la Ley General de Desarrollo Forestal Sustentable (LGDFS) y del reglamento que la rige, el bosque ribereño podría interpretarse como un ecosistema hidrológico-forestal y por tanto quedar sujeto a sus disposiciones, incluso en aquellos sitios donde la vegetación se haya eliminado parcial o totalmente. Por otra parte, la Ley General del Equilibrio Ecológico y Protección al Ambiente (LGEEPA) regula la preservación y restauración de la cobertura vegetal original (Tabla S1), y establece diversos instrumentos de política ambiental relevantes para la conservación y restauración de los bosques ribereños. Adicionalmente, existen dos Normas Oficiales Mexicanas (060 y 062; Tabla S1) que definen la vegetación ribereña como aquella que "crece sobre o cerca de los bancos de corrientes o cuerpos de agua en suelos que presentan ciertas características de humedad". Sin embargo, estas normas regulan la mitigación de los efectos ocasionados en los suelos, los cuerpos de agua y la biodiversidad por el aprovechamiento y el cambio de uso del suelo, y no en la vegetación ribereña per se.

Los marcos normativos antes mencionados se centran principalmente en la mitigación de los impactos negativos de los cambios de uso del suelo en suelos y cuerpos de agua, o incluso bosques, pero no están explícitamente definidos para los bosques ribereños como un tipo de ecosistema (Meli y Brancalion, 2017). Actualmente, la Ley General de Cambio Climático de 2012 avanzó la promoción oficial de la restauración en el país para mitigar y adaptarse al cambio climático al incluir el diseño de las políticas y los mecanismos para la protección, conservación y restauración de la vegetación de ribera de las zonas federales (art. 34), de conformidad con la Ley de Aguas Nacionales. Esta Ley contiene también otros conceptos clave para la restauración, como por ejemplo la única definición dentro del sistema legal mexicano de 'corredores biológicos', como un elemento del paisaje para la conectividad de los procesos biológicos y evitar el aislamiento de las poblaciones.

Sin embargo, de manera general, los instrumentos jurídicos para la conservación y restauración de los bosques ribereños en México todavía resultan poco sólidos, están dispersos y tienen omisiones. Su vaga definición y regulación se presta a ambigüedades. Sin embargo, algunos instrumentos de política pública serían útiles para poner en funcionamiento las acciones definidas en este marco (Tabla S1). Es necesaria la revisión del marco jurídico relacionado con los bosques ribereños en México, en especial para que especifiquen explícitamente la obligación de conservar y restaurar la vegetación ribereña en zonas federales, cuyo sustento legal podría ser la LGDFS (Ruiz, 2011). Al mismo tiempo, se pueden considerar instrumentos de política dirigidos a las áreas ribereñas fuera de las zonas federales para constituir zonas de amortiguamiento donde se permita introducir especies ecológicamente adecuadas que pueden ser mezcladas con otras útiles para el beneficio económico de los dueños de la tierra (ver apartado socioeconómico).

Un estudio sobre la percepción de la población local de los bosques ribereños en Marqués de Comillas mostró que los campesinos reconocen la importancia de la vegetación ribereña para el mantenimiento de sus cultivos y para evitar la erosión de sus tierras y que las poblaciones desconocen o tienen una idea vaga de la legislación referente al tema (Ruiz, 2011). Existen algunos acuerdos ejidales que plantean la necesidad de conservar las riberas; pero estos son verbales y en la mayor parte de los casos no existen multas $\mathrm{u}$ otras penas para aquellas personas que los incumplan (Meli et al., 2015c). Esta situación evidencia la necesidad de promover acuerdos escritos que exijan la conservación de la vegetación ribereña y su restauración en casos de degradación, y que establezcan responsabilidades en torno a su uso reflejando los intereses locales de las necesidades productivas y de uso del territorio. Estos reglamentos deberían desarrollarse en el marco de un contexto participativo, donde los pobladores locales deberían ser el elemento más importante. 


\section{DISCUSIÓN}

La evaluación de estos aspectos de la restauración de bosques ribereños destaca la necesidad de una aproximación integrada a la problemática de su conservación y recuperación. La plantación de árboles nativos es la herramienta más utilizada para restaurar la vegetación, pero debe ser combinada con otras opciones (siembra directa y conservación de suelos, entre otras; Rodrigues et al., 2009). Si bien la evaluación de los aspectos ecológicos de la restauración de bosques ribereños no es exhaustiva, sus resultados son promisorios. Aún queda el desafío de reducir los costos económicos, planear acciones a escala de paisaje y resolver cuestiones sociopolíticas para lograr una restauración exitosa en la región y poder hacer recomendaciones más sólidas para la restauración de bosques en los trópicos de México. Estos aspectos pueden ser las barreras más difíciles de superar. Un desafío de la restauración es que, con pocas excepciones (Bullock et al., 2011), los costos de diferentes técnicas utilizadas no suelen ser incluidos en la literatura (Wortley, Hero y Howes, 2013), lo que resulta en la carencia de información confiable para evaluar la rentabilidad de la restauración (Kimball et al., 2015). Los métodos más efectivos en la relación beneficio-costo (e. g. aquellos que resultan en mayor cobertura vegetal nativa por recurso económico invertido) no son necesariamente los que resultan en la mayor cobertura independientemente del costo económico (Suding et al., 2014).

La restauración de los bosques ribereños debería abordarse tanto a escala local como del paisaje y, de esta forma, podría enmarcarse en un contexto de manejo de cuencas, con criterios que permitan definir aquellas áreas donde la relación beneficio-costo de la restauración sea óptima (Meli et al., 2015a). Una valoración de la conectividad del paisaje a través de los bosques ribereños como corredores podría ayudar a identificar áreas críticas para la restauración, maximizando la relación beneficio-costo en los aspectos ecológico y social (Meli et al., 2015c). La discusión con las comunidades locales permitirá ajustar una estrategia regional; sin embargo, debe continuarse la investigación a escala local para afinar la posible aplicación de estos modelos a esta escala. Al mismo tiempo, aún falta trabajo relacionado con algunas limitaciones técnicas (p. ej. los costos de mantenimiento) y sociales (p. ej. la aceptación social y la incorporación de la restauración ecológica en programas públicos) para ser aplicados a escala regional o de microcuenca, así como una estimación directa de los servicios ecosistémicos que podrían restablecerse a partir de la restauración de riberas. Esto puede afectar directamente la cantidad ofertada de servicios ecosistémicos ya que controla los patrones y las tasas tanto de los flujos bióticos (p. ej. dispersión, movimiento de fauna) como de los abióticos (p. ej. ciclos biogeoquímicos y de nutrientes), pero también se ve indirectamente afectado por la alteración de los niveles de biodiversidad y del funcionamiento de los ecosistemas (Mitchell, Bennet y González, 2013). Existen pocos ejemplos que consideran una selección de servicios ecosistémicos de una manera integrada (Bullock et al., 2011).

La conservación, manejo y restauración de los bosques ribereños, su biodiversidad y servicios en México debería llevarse a cabo en el contexto de una política transversal de gestión desde diversos sectores, comprendiendo los aspectos ecológicos, sociales y económicos, y orientada hacia tres líneas estratégicas: conservación, uso y restauración (Tabla 2). Estas recomendaciones podrían adaptarse, en especial en materia de política pública, a dos condiciones particulares y complementarias, es decir, (1) medidas exclusivamente de conservación dirigidas a una franja de dominio público y (2) medidas relacionadas con el manejo sustentable en las franjas de amortiguamiento, con regímenes parcelario, privado o común, dependiendo de la región. De esta manera, aunque tal vez no se lograra regresar a las condiciones prístinas, la restauración de bosques ribereños podría enmarcase en un contexto de consenso, donde se combinaran los beneficios a los pobladores locales junto con la recuperación del ecosistema.

\section{CONCLUSIONES}

La restauración de bosques ribereños debe basarse en la investigación, pero al mismo tiempo deber proporcionar soluciones realizables que guíen mejores prácticas apro- 
TABLA 2. Recomendaciones relacionadas con la conservación, manejo y restauración de los bosques ribereños.

\begin{tabular}{|c|c|c|c|}
\hline & Recomendaciones generales & $\begin{array}{c}\text { Líneas } \\
\text { estratégicas }\end{array}$ & Recomendaciones específicas \\
\hline \multirow{3}{*}{ 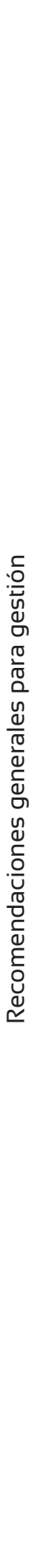 } & $\begin{array}{l}\text { Incorporar la definición de vegetación ribereña } \\
\text { en las leyes federales o nacionales (en parti- } \\
\text { cular en la Ley General de Desarrollo Forestal } \\
\text { Sustentable). } \\
\text { Incluir las riberas como ecosistemas espe- } \\
\text { cíficos en los programas de conservación, } \\
\text { manejo y restauración tanto gubernamentales } \\
\text { como privados. } \\
\text { Incluir las riberas como ecosistemas específ- } \\
\text { cos en los instrumentos de gestión ambiental } \\
\text { o de manejo de recursos (p.ej. ordenamiento } \\
\text { territorial, sistemas agrosilvopastoriles, corre- } \\
\text { dores biológicos). } \\
\text { Promover el manejo de cuencas y el manejo }\end{array}$ & 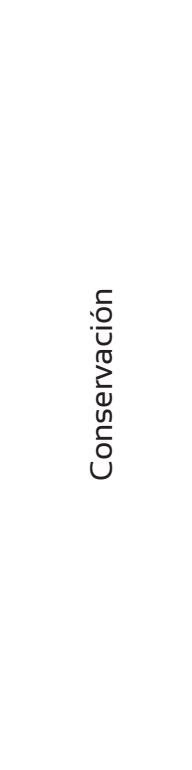 & $\begin{array}{l}\text { Deslindar los bosques ribereños como zonas federales o } \\
\text { de interés público. } \\
\text { Revisar el ancho mínimo necesario para mantener las } \\
\text { funciones de los bosques ribereños y de los ecosistemas } \\
\text { acuáticos asociados. } \\
\text { Conservar áreas de captación en las microcuencas. } \\
\text { Implementar incentivos para la conservación de riberas en } \\
\text { el marco del manejo sustentable de los recursos natura- } \\
\text { les. } \\
\text { Revisar la normativa legal relacionada con el manejo y uso } \\
\text { de los bosques ribereños. } \\
\text { Difundir la legislación ambiental. } \\
\text { Identificar ecosistemas de referencia y especies poten- } \\
\text { cialmente útiles. }\end{array}$ \\
\hline & $\begin{array}{l}\text { integrado del recurso hídrico a escala local y } \\
\text { regional. } \\
\text { Establecer corredores biológicos ribereños en } \\
\text { el marco de programas institucionales. } \\
\text { Promover la cooperación institucional. } \\
\text { Evaluar las percepciones sociales de los dis- } \\
\text { tintos actores para buscar consensos. } \\
\text { Monitorear los procesos funcionales en el } \\
\text { ecosistema. } \\
\text { Promover la educación ambiental y la partici- } \\
\text { pación social. }\end{array}$ & 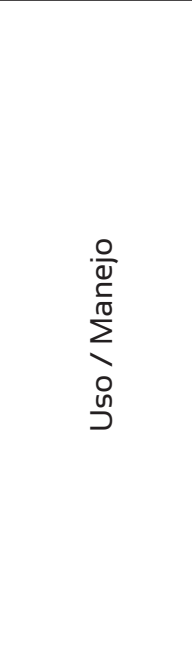 & $\begin{array}{l}\text { Revegetar franjas de amortiguamiento, constituidas por } \\
\text { especies útiles en riberas conservadas o de bajo uso. } \\
\text { Promover reglamentos de conservación, manejo y restau- } \\
\text { ración de riberas a escalas locales, microregionales o de } \\
\text { cuenca. } \\
\text { Promover el manejo sustentable y productivo de los eco- } \\
\text { sistemas acuáticos asociados a las riberas. } \\
\text { Poner en marcha las distintas prácticas de conservación } \\
\text { de suelos en las riberas. } \\
\text { Incluir las riberas como un componente específico de } \\
\text { los sistemas productivos (en México, particularmente los } \\
\text { sistemas agrosilvopastoriles). }\end{array}$ \\
\hline & & 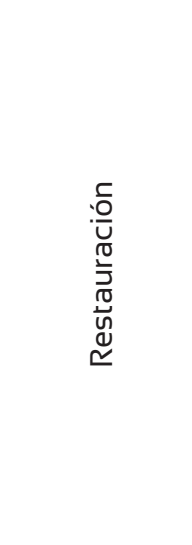 & $\begin{array}{l}\text { Recuperar los suelos y realizar obras de conservación en } \\
\text { caso de erosión física grave. } \\
\text { Introducir vegetación herbácea con crecimiento subte- } \\
\text { rráneo en áreas con procesos avanzados de erosión del } \\
\text { suelo. } \\
\text { Introducir árboles nativos en áreas abiertas (revegetación). } \\
\text { Enriquecer con especies nativas las riberas con regenera- } \\
\text { ción secundaria. } \\
\text { Controlar el uso de agroquímicos y la descarga de resi- } \\
\text { duos tóxicos a los cuerpos de agua. }\end{array}$ \\
\hline
\end{tabular}


piadas de acuerdo con los marcos legales, políticos e institucionales (Suding et al., 2014). Esto sería más coherente con las ciencias ecológicas y sociales y con la mayor probabilidad de realizar una restauración que brindara beneficios ecológicos y económicos con una mayor aceptación social. Aún existen dificultades para traducir las variables sociales que afectan los procesos ecológicos, especialmente en regiones pobres en datos o aquellas con problemática social compleja.

Resulta esencial centrarse en investigaciones empíricas sobre la cuantificación de los servicios ecosistémicos que brindan los bosques ribereños para comprender los beneficios y costos totales de la restauración ecológica de estos ecosistemas. Una correcta y amplia valoración de la relación beneficio-costo de las acciones de restauración es fundamental para asegurar que los fondos limitados se gasten de la mejor manera y para evitar gastar dinero en acciones ineficaces (Birch et al., 2011). A pesar de los posibles compromisos entre la viabilidad social y los beneficios ecológicos de la restauración, la integración de los aspectos ecológicos, socioeconómicos y legales ayuda a la integración para dar lugar a soluciones más realistas y factibles.

\section{RECONOCIMIENTOS}

El presente trabajo fue desarrollado dentro del "Programa de restauración ambiental en la región de Marqués de Comillas para favorecer la conservación de la selva y aumentar la conectividad del paisaje”, que desarrolló Natura y Ecosistemas Mexicanos A.C. con el financiamiento de PEMEX, la Alianza WWF-FCS. PM recibió apoyo de la Fundación Rufford (Small Grant for Nature Conservation 40.11.09). Los autores agradecen el apoyo de los estudiantes de la Facultad de Ciencias de la UNAM que desarrollaron su servicio social en Marqués de Comillas y de los trabajadores de la Estación Chajul.

\section{REFERENCIAS}

Aguilar-Fernández, R. (2013). Análisis de los componentes socioambientales para la restauración de claros antropogénicos en la selva tropical húmeda, en el municipio de Marqués de Comillas, Chiapas. Tesis de Maestría, Universidad Nacional Autónoma de México, México D.F.

Allen, A. E., Santana-Michel, F. J., Ortiz Arrona, C. y Zedler, J. B. (2010). Integrating ecological and ethnobotanical priorities into riparian restoration. Ecological Restoration, 28, 377-388.

Birch, J., Newton, A. C., Alvarez-Aquino, C., Cantarello, E., Echeverría, C., Kitzberger, T., Schiappacasse, I. y Tejedor-Garavito, N. (2010) Cost-effectiveness of dryland forest restoration evaluated by spatial analysis of ecosystem services. Proceedings of the National Academy of Sciences U.S.A., 107, 21925-21930.

Budiharta, S., Meijaarda, E., Wells, J. A., Abram, N. A. y Wilson, K. A. (2016). Enhancing feasibility: Incorporating a socio-ecological systems framework into restoration planning. Environmental Science \& Policy, 64, 83-92.

Bullock, J. M., Aronson, J., Newton, A. C., Pywell, R. F. y Rey Benayas, J. M. (2011). Restoration of ecosystem services and biodiversity: conflicts and opportunities. Trends in Ecology and Evolution, 26, 541-549.

Castro, E. y Ortiz, F. (2015). Ordenamiento comunitario del territorio. En J. Carabias, J. de la Maza y R. Cadena (Coord.). Conservación y desarrollo sustentable en la Selva Lacandona. 25 años de actividades y experiencias (pp. 471-486), México D.F., México: Natura y Ecosistemas Mexicanos A.C.

Ceccon, E. y Pérez, D. R. (coord.) (2016). Más allá de la ecología de la restauración: perspectivas sociales en América Latina y el Caribe. Buenos Aires, Argentina: Vázquez Mazzini Editores.

Centro Interdisciplinario de Biodiversidad y Ambiente (2010). Aplicación de mecanismos de reducción de emisiones por deforestación y degradación (REDD) en ejidos y comunidades. México D.F., México: Programa de Competitividad de la USAID/México.

Comisión Nacional de Salarios Mínimos (2015). Nuevos salarios mínimos por área geográfica 2013. Recuperado de www.conasami.gob.mx/nvos_sal_2013.html

Echeverría, C., Smith-Ramírez, C., Aronson, J. y BarreraCataño, J. I. (2015). Establishing the ecological restora- 
tion network in Argentina: from Rio1992 to SIACRE2015. Restoration Ecology, 23, 95-103.

Grupo Autónomo para la Investigación Ambiental, A.C. (Gaia) y Natura y Ecosistemas Mexicanos A.C. (Natura). (2012). Ordenamiento comunitario del territorio de la microrregión Marqués de Comillas, Chiapas. México D.F., México: Gaia, Natura y Comisión Nacional Forestal.

Higgs, E., Falk, D. A., Guerrini, A., Hall, M., Harris, J., Hobbs, R. J., Jackson, S. T., Rhemtulla, J. M. y Throop, W. (2014). The changing role of history in restoration ecology. Frontiers in Ecology and the Environment, 12, 499-506.

Hobbs, R. J., Davis, M. A., Slobodkin, L. B., Lackey, R. T., Halvorson, W., Throop, W. (2004). Restoration ecology: The challenge of social values and expectations. Frontiers in Ecology and the Environment, 2, 43-48.

Holl, K. D. y Crone, E. E. (2004). Applicability of landscape and island biogeography theory to restoration of riparian understory plants. Journal of Applied Ecology, 41, 922933.

Instituto Nacional de Ecología (2000). Programa de manejo reserva de la Biósfera Montes Azules México. México D.F., México: Instituto Nacional de Ecología.

Instituto Nacional de Estadística y Geografía (2010). XIII Censo general de población y vivienda. México: Instituto Nacional de Estadística y Geografía.

Kimball, S., Lulow, M., Sorenson, Q., Balazs, K., Fang, Y. C, Davis, S. J., O’Connell, M. y Huxman, T. E. (2015). Costeffective ecological restoration. Restoration Ecology, 23, 800-810.

Kondolf, G. M., Angermeier, P. L., Cummins, K., Dunne, T., Healey, M., Kimmerer, W., Moyle, P. B., Murphy, D. Patten, D., Railsback, S., Reed, D. J., Spies, R., Twiss, R. (2008). Projecting cumulative benefits of multiple river restoration projects: an example from the Sacramento San Joaquin River system in California. Environmental Management, 42, 933-945.

Martínez Ramos, M. y García Orth, X. (2007). Sucesión ecológica y restauración de las selvas húmedas. Boletín de la Sociedad Botánica de México, 80, 69-84.

Martínez Ramos, M., Barraza, L., Balvanera, P. et al. (2012). Manejo de bosques tropicales: bases científicas para la con- servación, restauración y aprovechamiento de ecosistemas en paisajes rurales. Investigación Ambiental, 4, 111-129.

McDonald, T., Gann, G. D., Jonson, K. y Dixon, K. W. (2016). International standards for the practice of ecological restoration - including principles and key concepts. Washington, D.C., USA: Society for Ecological Restoration.

Meli, P., Martínez-Ramos, M. y Rey-Benayas, J. M. (2013). Selecting species for passive and active riparian restoration in Southern Mexico. Restoration Ecology, 21, 163 165.

Meli, P., Rey Benayas, J. M., Carabias, J., Ruiz, L. y MartínezRamos, M. (2013). Restauración de los servicios ecosistémicos ribereños. Meta-análisis global y un estudio de caso en Chiapas, México. En A. Lara, P. Laterra, R. Manson y G. Barrantes (Eds.). Servicios ecosistémicos hídricos en América Latina y el Caribe (pp. 39-58). Valdivia, Chile: Red ProAgua - CYTED.

Meli, P., Martínez-Ramos, M., Rey Benayas, J. M. y Carabias, J. (2014). Combining ecological, social, and technical criteria to select species for forest restoration. Applied Vegetation Science, 17, 744-753.

Meli, P., Aguilar-Fernández, R. y Carabias, J. (2015a). Restauración ecológica en Marqués de Comillas. En J. Carabias, J. de la Maza y R. Cadena (Coord.). Conservación y desarrollo sustentable en la Selva Lacandona. 25 años de actividades y experiencias (pp. 429-458), México D.F., México: Natura y Ecosistemas Mexicanos A.C.

Meli, P., Rey-Benayas, J. M., Martínez-Ramos, M. y Carabias, J. (2015b). Effects of grass clearing and soil tilling on establishment of planted tree seedlings in tropical riparian pastures. New Forest, 6, 507-525.

Meli, P., Hernández-Cárdenas, G., Castro, E. y Carabias, J. (2015c). Vinculando paisaje y parcela: un enfoque multiescala para la restauración ecológica en áreas rurales. Investigación Ambiental, 7, 43-53.

Meli, P., Landa, R., López-Medellín, X. y Carabias, J. (2015d). Social perceptions of rainforest and climatic change from rural communities in Southern Mexico. Ecosystems, 18, 1343-1355.

Meli, P. y Brancalion, P.H.S. (2017). Contrasting regulatory frameworks to govern riparian forest restoration in Mexico 
and Brazil: Current status and needs for advances. World Development Perspectives, 5, 60-62.

Mitchell, M. G. E., Bennet, E. M. y Gonzalez, A. (2013). Linking landscape connectivity and ecosystem service provision: current knowledge and research gaps. Landscape Ecology, 16, 894-908.

Natura y Ecosistemas Mexicanos A.C. (Natura) (2013). Restauración ambiental en la región de Marqués de Comillas para favorecer la conservación de la selva y aumentar la conectividad del paisaje. Informe Anual - PEMEX, México.

Ortiz, F., Rubio, N., Carabias, J. y Vázquez, P. (2015). Pago por servicios ambientales. En J. Carabias, J. de la Maza y R. Cadena (Coord.). Conservación y desarrollo sustentable en la Selva Lacandona. 25 años de actividades y experiencias (pp. 315-332), México D.F, México: Natura y Ecosistemas Mexicanos A.C.

Ramírez Carrillo, E. (2006). Evaluación de la diversidad florística de la vegetación ribereña bajo diferentes condiciones de conservación en la Selva Lacandona: hábitat crítico para la guacamaya roja. Tesis de Maestría, Universidad Nacional Autónoma de México, México D.F., México.

Ramírez-Martínez, C. (2010). Efectos de la desaparición de la selva sobre ambientes acuáticos. En S. Chediack (Comp.). Monitoreo de biodiversidad y recursos naturales ¿para qué? Serie Diálogos/Número 3, Colección Corredor Biológico Mesoamericano, México D.F., México: Comisión Nacional para el Conocimiento y Uso de la Biodiversidad, $87 \mathrm{pp}$.

Rodrigues, R. R., Lima, R. A. F., Gandolfi, S. y Nave, A. G. (2009). On the restoration of high diversity forests: 30 years of experience in the Brazilian Atlantic Forest. Biological Conservation, 142, 1242-1251.

Román-Dañobeytia, F. J., Levy-Tacher, S., Aronson, J., RibeiroRodrigues, R. y Albores, J. (2012). Testing the performance of fourteen native tropical tree species in two abandoned pastures of the Lacandon rainforest region of Chiapas, Mexico. Restoration Ecology, 20, 378-386.

Ruiz, L. (2011). Herramientas legales para la conservación y restauración de la vegetación ribereña: un estudio de caso en la Selva Lacandona. Tesis de Licenciatura, Universi- dad Nacional Autónoma de México, México D.F., México.

Sabo, J. L., Sponseller, R., Dixon, M., Gade, D., Harms, T., Heffernan, J., Jani, A., Katz, G., Soykan, C., Watts, J. y Welter, J. (2005). Riparian zones increase regional species diversity by harboring different, not more species. Eco$\log y, 86,56-62$.

Soares-Filho, B., Rajão, R., Macedo, M., Carneiro, A., Costa, W., Coe, M., Rodrigues, H. y Alencar, A. (2014). Cracking Brazil's Forest Code. Science, 344, 363-364.

Society for Ecological Restoration (SER) (2004). Principios de SER International sobre la restauración ecológica. Recuperado de http://www.ser.org/docs/default-documentlibrary/spanish.pdf.

Suding, K., Higgs, E., Palmer, M., Callicott, J. B., Anderson, J. C., Baker, M., Gutrich, J. J., Hondula, K. L., LaFevor, M. C., Larson, B. M. H., Randall, A., Ruhl, J. B. y Schwartz, K. Z. S. (2014). Committing to ecological restoration. Science, 348(6235), 638-640.

Watanabe, M., Adams, R. M., Wu, J., Bolte, J. P., Cox, M. M., Jonhson, S. L., Liss, W. J., Bogges, G. y Ebersole, J. L. (2005). Toward efficient riparian restoration: integrating economic, physical, and biological models. Journal of Environmental Management, 75, 93-104.

Weisberg, P. J., Mortenson, S. G. y Dilts, T. E. (2013). Gallery forest or herbaceous wetland? The need for multi-target perspectives in riparian restoration planning. Restoration Ecology, 21, 122-16.

Wilson, A., Jansen, A., Curtis, A. y Robertson, A. (2006). Measuring riparian condition: a comparison of assessments by landholders and scientists. Ecological Management and Restoration, 7, 123-129.

Wortley, L., Hero, J. M. y Howes, M. (2013). Evaluating Ecological Restoration Success: A Review of the Literature. Restoration Ecology, 21, 537-543.

Manuscrito recibido el 7 de agosto de 2016.

Aceptado el 24 de enero de 2017.

Este documento se debe citar como:

Meli, P., Ruiz, L., Aguilar, R., Rabasa, A., Rey-Benayas, J. M. y Carabias, J. (2017). Bosques ribereños del trópico húmedo de México: un caso de estudio y aspectos críticos para una restauración exitosa. Madera y Bosques, 23(1), 181-193. doi: 10.21829/myb.2017.2311118 